\title{
Energy Efficient Mobile Element Based Data Gathering in Wireless Sensor Networks
}

\author{
Mr.P.Shanmugam ${ }^{1}$, Ms.S.Jayanthi ${ }^{2}$, Dr.J.Raja ${ }^{3}$, Ms.R.Nusrath ${ }^{4}$ \\ Asst.Professor, Department of Information Technology, Adhiparasakthi Engineering College, Melmaruvathur, India ${ }^{1}$ \\ ME Communication and Networking, Adhiparasakthi Engineering College, Melmaruvathur, India ${ }^{2,4}$ \\ Professor, Department of IT, Adhiparasakthi Engineering College, Melmaruvathur, India ${ }^{3}$
}

\begin{abstract}
In recent years, Data Gathering plays an important role in wireless sensor network. The data can be gathered using two methods. First, Static Sink can be used to collect data from sensors and routed to mobile station via multi hop communication. This increases the delay and consumes high energy. To avoid this problem, a mobile element based data gathering is introduced. The ME has a shortest path and it can move along the path and collects data from sensors and passes the data to the Mobile station. This reduces the delay but ME depletes its energy often. To reduce the consumption of energy, Energy Efficient mobile element based data gathering using Tinybee is proposed. In this method, the tinybees are dispatched from the mobile element and comes back to the ME with aggregated data. So, the movement of ME is reduced. Thus it reduces the tour length of the ME and energy can be efficiently utilized. This proposed method achieves the energy efficiency to an acceptable level.
\end{abstract}

Keywords: Wireless sensor Networks, Tinybee, Mobile Element

\section{INTRODUCTION}

Recently, Wireless sensor network is an emerging platform which has an attention from the research. Sensor network is a self-organizing and sensors are distributed over a large or small sensor network. It can monitor physical, biological environment to gather information and passes the gathered information to the base station.

Each sensor node contains various components such as transceiver, external memory, micro-controller, battery, Analog to Digital converter and Sensing unit. The sensing unit senses the physical condition of the environment and passes the information to the ADC and it in turn converts the analog data to digital data. Then micro controllers process the sensed data and passes to transceiver to transmit the data. It can also receive the data from the environment.

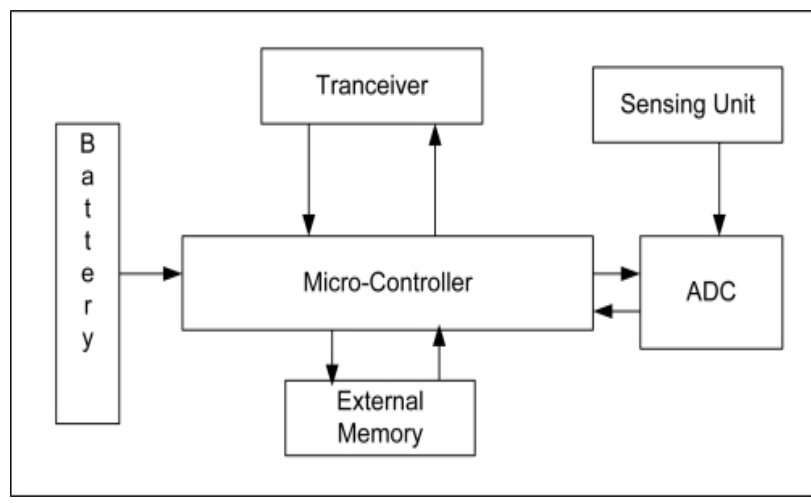

Fig. 1 Sensor Architecture

The sensor network has variety of applications includes medical environment, industrial environment, physical environment and biological environment. It can be supported for both real time and non-real time applications.

There are three stages in data gathering: sensing, processing and transferring. The sensing unit monitors and senses the data from the location the sensor is deployed and then the data can be processed to avoid the redundancy and at last transfers the data to the base station. The data can be relayed using two methods: (1) Single-hop communication (2) Multi-hop Communication.
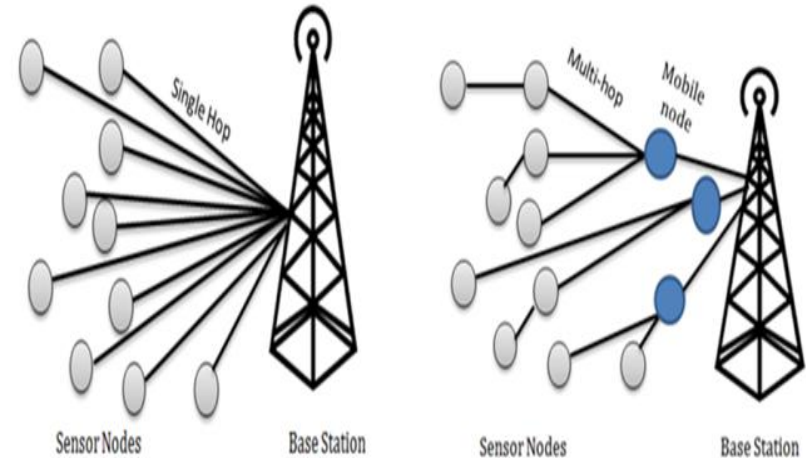

Fig. 2 Single hop and Multi-hop Communication

\section{A. Issues of Data Gathering in WSN}

1)The mobile collector is a dynamic node that acts as an intermediate node to deliver data from sensor nodes to base station BS. The mobile collector collects data from sensors and passes to BS. This causes delay in data gathering.

2)In WSN, Efficient usage of energy is a major problem. The sensor network consists of plenty of sensors which can be deployed in the deployment field. It consumes more energy. The efficient usage of energy is essential.

3)Large amount of data can be gathered simultaneously from multiple sensors can reduce the energy consumption which is a big task in WSN. Thus the balance among these two is necessary. 
4)In battlefield application, the base station is far away from the mobile collector. Thus the delay is increased for passing data from mobile collector to BS.

5) The sensor nodes have limited battery life. So, the sensor nodes have to be recharged or changed frequently. Thus it consumes more energy.

\section{B. Limitations in WSN}

1)Hostile Environment:Sensor networks can be placed in hostile environments such as battlefields. In these cases, the nodes cannot be protected from physical attacks, since anyone can access to the location where they are deployed. An opponent could capture sensor node or even introduce his own malicious nodes inside the network. Thus, the opponent's aim is to hack the sensor network.

2)Random Topology:Deploying a sensor network in a hostile environment is done by random distribution, i.e. from an airplane. Thus, it is difficult to know the topology of sensor networks a priori.

3)Limited Resources:

- Power Limitations:The Sensor nodes are normally battery-dependent. A sensor network contains hundreds to thousands of nodes, and deployed in remote or hostile environments, it is difficult to replace or boost batteries. The excess power is consumed for processing the information gathered and for communication.

- Storage Restrictions: The sensor nodes have to gather data from the environment such as temperature, humidity, sound, etc. and store it. The storage capacity of nodes is limited. This causes buffer overflow.

\section{Challenges of Data Gathering in WSN}

1)The sensor network routes data from sensor node to base station depending on network topology.

2)The same path can be used by multiple sensors to pass data to BS which in turn saves energy without selecting alternate path.

3)Multiple sensors send data to base station simultaneously increases data loss and retransmission consumes high energy and maximize the delay.

\section{RELATED WORK}

AnK.Ramanan and E.Baburaj[1] outlined different serious issues in wireless sensor network. A general study of different issues associated with existing data gathering algorithms is done and two key issues are focused. The issues focused on: network lifetime and energy saving. In a Wireless sensor networks, data can be collected by the sinks by extending the network lifetime. Hence the sink collects multiple data. The efficient usage of energy is serious for the networks lifetime.

Mario Di Francesco et al [2] have extensively categorized data collection in Wireless Sensor Networks with Mobile Elements (WSN-MEs). An overview of the data collection process is presented and the corresponding issues and challenges are identified. The data collected from the sensors is again collected by the mobile elements. This reduces the energy consumption.
Feng Wang and JiangchuanLiu[3] have presented a survey on the usage of WSNs for sensor data collection. The different approaches for control message dissemination are discussed. It acts as an essential component for network control and management. It can also affect the overall performance of WSNs for sensor data collections.

Ramesh Rajagopalan and Pramod K. Varshney[4] have presented a comprehensive survey of data aggregation algorithms in wireless sensor networks. Most of the works focus on optimization of important performance measures such as network lifetime, data latency, data accuracy and energy consumption. The three main focus areas of data aggregation algorithms are efficient organization, routing and data aggregation tree construction.

Kaoru Ota, Mianxiong Dong [5] proposed Tinybee: a mobile agent based data gathering technique in WSN. Tinybee can be used to gather data over a network. Tinybees moves node to node after being dispatched from the mobile server in order to collect data from sensors. So the movement of mobile sensors are reduced. This model is both time efficient and energy efficient.

\section{III.PROPOSED WORK}

The proposed work explains energy efficient mobile element based data gathering approach using tinybee in wireless sensor networks.In this method, the sensors are deployed in a deployment field. The sensor senses the data and processes the gathered data and aggregated the data. The Mobile element can be used to gather the aggregated data from the sensors. If the mobile elements needs to travel to each sensors to gather data then the delay will be increased. Thus The ME dispatches the tinybee in order to collect data from sensors. After collecting the data from sensors, it comes back to the ME with accumulated data. So, there is a reduction movement of ME. Thus it reduces the tour length of the ME and energy can be efficiently utilized.

Four phases are included for data gathering from the sensor node (1) Sensor node deployment (2) Registration of path of mobile element (3) Dispatching the Tinybee (4) Data gathering.

\section{Sensor Node Deployment}

The sensor nodes are deployed in the deployment field. If it deploys in a random manner then the intruder can hack the data during the transmission of data from sensor to mobile element. The proposed system uses a normal distribution to deploy the sensor in the network. This reduces the retrieval of data during transmission. The sensor node can be deployed in any region including volcanic region, hilly region, desert, etc. The sensors can be deployed based on the needs to be satisfied. The sensor can be of any type according to the type of data to be gathered.

\section{E. Registration of path of mobile element}

If it needs to gather data from each sensor individually, then the delay is increased and energy cannot be efficiently used. The energy consumption of sensor nodes is limited. Thus the energy of sensor node is depleted 
frequently. So the path of the mobile element is registered mobile element. The proposed work increases the network to reduce the tour length of the ME. Thus the mobile element can passes along the registered path to dispatch the tinybee. This reduces the delay and the energy of ME can be reduced.

\section{F. Dispatching the tinybee}

The Mobile element dispatches the tinybee when passes along the registered path. The tinybee spreads over the network and takes a place in the network. It collects data from the neighbourhood sensors.

After collecting the data, the tinybee moves towards the mobile element by using the convergent node. The convergent keeps track of all the tinybees and its location. The aggregated data is then sent to the mobile element.

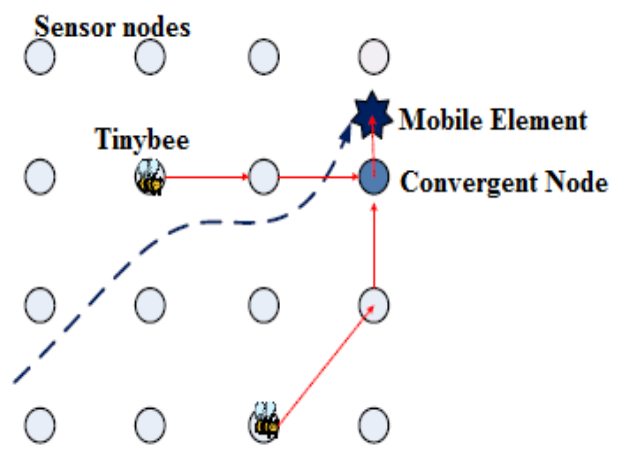

Fig. 3 Dispatching of tinybee by mobile element

\section{G. Data Aggregation}

The mobile element gathers the data from all tinybees and aggregates the data. The aggregated data is then sent to the Base station.

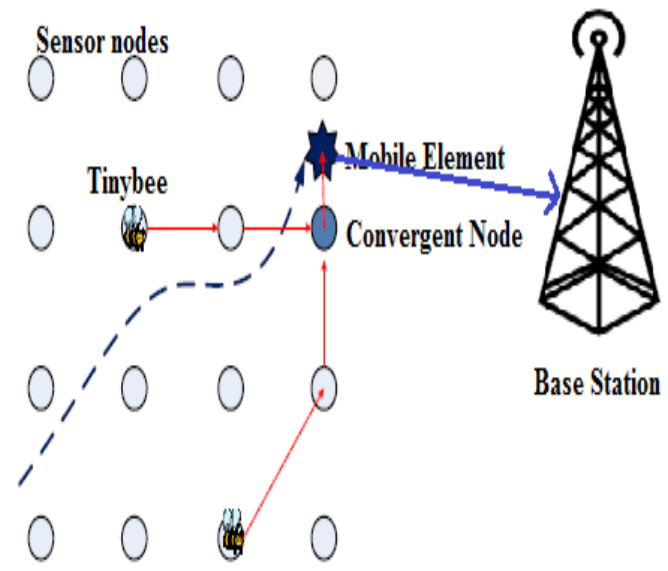

Fig. 4 Relaying data from mobile element to BS

\section{IV.PERFORMANCE EVALUATION}

In order to calculate the efficiency, the proposed work is implemented in 500X500 meter square region with 500 sensor nodes. The nodes are deployed based on binomial distribution in the deployment field.

The proposed scheme is compared with normal mobile element based on data gathering approach which reduces the data gathering latency and increases the network lifetime due to less energy utilization. The dispatching of tinybees by mobile element reduces the tour length of the

lifetime compared to existing schemes.

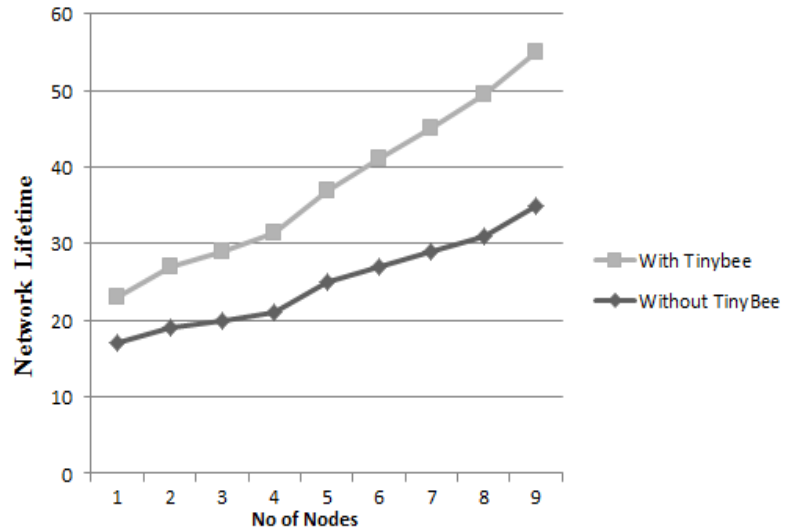

Fig. 5 Comparison of network lifetime with existing algorithms

The proposed algorithm utilizes less energy than existing work. After collecting data from sensors, the tinybees segregates the relevant and irrelevant data. After segregation, it aggregates the data and passes it to the mobile element. This reduces the drop rate of gathered data during transmission.

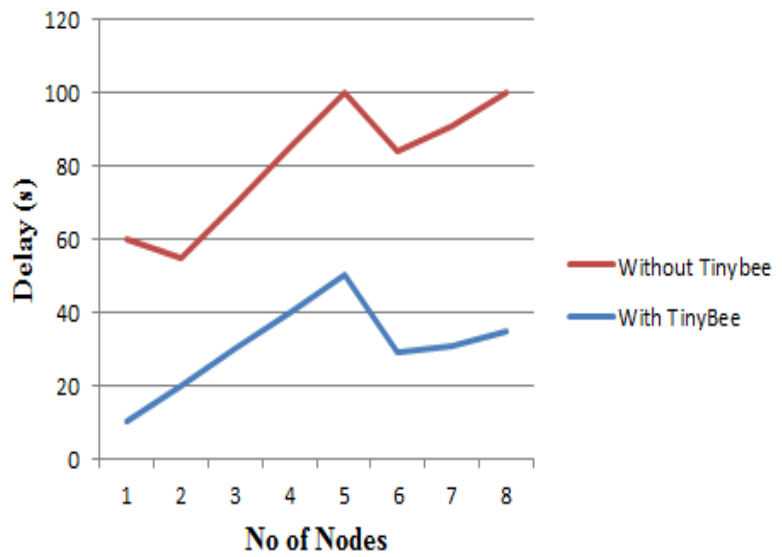

Fig. 6 Comparison of delay with existing algorithm

\section{CONCLUSION AND FUTURE ENHANCEMENT}

In the proposed scheme, energy efficient mobile element based data gathering using tinybee achieves energy efficiently and reduces the latency in data gathering. The dispatch of tinybees by mobile element reduces the tour length of the Mobile element. The proposed work is determined based on delay and energy. The proposed work achieves increased network lifetime due to less energy utilized than other algorithms. The future enhancement is to apply multiple mobile elements which in turn dispatch multiple tinybees for efficient gathering of data in large sensor network.

\section{REFERENCES}

[1] K.Ramanan and E.Baburaj, "Data Gathering Algorithms For Wireless Sensor Networks: A Survey", International Journal of Ad hoc, Sensor \& Ubiquitous Computing (IJASUC) Vol.1, No.4, December 2010, pp.102-114.

[2] Mario Di Francesco, Sajal K. Das, Giuseppe Anastasi "Data Collection in Wireless Sensor Networks with Mobile Elements: A Survey", Journal on ACM Transactions on Sensor Networks (TOSN), August 2011, Vol. 8, Issue 1, , Article No. 7. 
[3] Feng Wang and Jiangchuan Liu, "Networked Wireless Sensor Data Collection: Issues, Challenges, and Approaches", IEEE Communications Surveys \& Tutorials For Possible Publication, 2011.

[4] Ramesh Rajagopalan and Pramod K. Varshney, "Data aggregation techniques in sensor networks: A survey", Communications Surveys \& Tutorials, IEEE, 2006, vol. 8, pp.48 - 66.

[5] Kaoru Ota, Mianxiong Dong, "TinyBee: Mobile-Agent-Based Data Gathering System in Wireless Sensor Networks".

[6] MukhtarGhaleb, ShamalaSubramaniam, Mohamed Othman and ZuriatiZukarnain, Predetermined path of mobile data gathering in wireless sensor networks based on network layout. EURASIP Journal on Wireless Communications and Networking 2014.

[7] A. Muthu Krishnan P. Ganesh Kumar, An Effective Clustering Approach with Data Aggregation Using Multiple Mobile Sinks for Heterogeneous WSN. Wireless Personnel Communication 2015.

[8] GopiSaminathanArumugam and ThirumuruganPonnuchamy EELEACH: development of energy-efficient LEACH Protocol for data gathering in WSN. EURASIP Journal on Wireless Communications and Networking 2015

[9] Wang Liu, Kejie Lu, Jianping Wang, Guoliang Xing and Liusheng Huang, Performance Analysis of Wireless Sensor Networks with Mobile Sinks. IEEE transactions on vehicular technology, vol. 61, No. 6, July 2012

[10] Jea, D., Somasundara, A., \&Srivastava M. B. (2005). Multiple controlled mobile elements (data mules) for data collection in sensor networks. In Proceedings of the 1st IEEE/ACM DCOSS.

[11] Wichmann, A., \&Korkmaz, T. (2015). Smooth path construction and adjustment for multiple mobile sinks in wireless sensor networks. Computer Communications. doi:10.1016/j.comcom.2015.06.001

[12] Shi, L., Zhang, B. X., Mouftah, H. T., \& Ma, J. (2013). DDRP: An efficient data-driven routing protocol for wireless sensor networks with mobile sinks. International Journal of Communication Systems, 26,1341-1355.

[13] Liu, W., Lu, K. J., Wang, J. P., Xing, G. L., \& Huang, L. S. (2012). Performance analysis of wireless sensor networks with mobile sinks. IEEE Transactions on Vehicular Technology, 61, 2777-2788.

[14] Lin, C. J. (2006). HCDD: Hierarchical cluster-based data dissemination in wireless sensor networks with mobile sink. In Proceedings of international conference on wireless communications and mobile computing, Vancouver, Canada (pp. 1189-1194).

[15] Shah, R. C., Roy, S., Jain, S., \& Brunette, W. (2003). Data MULEs: Modelinga three-tier architecture for sparse sensor networks. In Proceedings of IEEE workshop sensor network protocols and applications (SNPA).

[16] Konstantopoulos, C., Pantziou, G., Gavalas, D., Mpitziopoulos, A., \& Mamalis, B. (2012). A rendezvous- based approach enabling energy-efficient sensory data collection with mobile sinks. Parallel and Distributed Systems, IEEE Transactions on, 23(5), 809-817. 